\title{
Histone deacetylase 9 promoter hypomethylation associated with adipocyte dysfunction is a statin-related metabolic effect
}

Amna Khamis ${ }^{1,2}$, Raphael Boutry ${ }^{2}$, Mickaël Canouil ${ }^{2}$, Sumi Mathew ${ }^{1}$, Stephane Lobbens ${ }^{2}$, Hutokshi Crouch', Toby Andrew ${ }^{1}$, Amar Abderrahmani ${ }^{2}$, Filippo Tamanini ${ }^{1}$ and Philippe Froguel ${ }^{1,2^{*}}$ (D)

\begin{abstract}
Background: Adipogenesis, the process whereby preadipocytes differentiate into mature adipocytes, is crucial for maintaining metabolic homeostasis. Cholesterol-lowering statins increase type 2 diabetes (T2D) risk possibly by affecting adipogenesis and insulin resistance but the (epi)genetic mechanisms involved are unknown. Here, we characterised the effects of statin treatment on adipocyte differentiation using in vitro human preadipocyte cell model to identify putative effective genes.

Results: Statin treatment during adipocyte differentiation caused a reduction in key genes involved in adipogenesis, such as ADIPOQ, GLUT4 and ABCG1. Using Illumina's Infinium '850K' Methylation EPIC array, we found a significant hypomethylation of cg14566882, located in the promoter of the histone deacetylase 9 (HDAC9) gene, in response to two types of statins (atorvastatin and mevastatin), which correlates with an increased HDAC9 mRNA expression. We confirmed that HDAC9 is a transcriptional repressor of the cholesterol efflux ABCG1 gene expression, which is epigenetically modified in obesity and prediabetic states. Thus, we assessed the putative impact of ABCG1 knockdown in mimicking the effect of statin in adipogenesis. $A B C G 1 \mathrm{KD}$ reduced the expression of key genes involved in adipocyte differentiation and decreased insulin signalling and glucose uptake. In human blood cells from two cohorts, ABCG1 expression was impaired in response to statins, confirming that $A B C G 1$ is targeted in vivo by these drugs.

Conclusions: We identified an epigenetic link between adipogenesis and adipose tissue insulin resistance in the context of T2D risk associated with statin use, which has important implications as HDAC9 and ABCG1 are considered potential therapeutic targets for obesity and metabolic diseases.
\end{abstract}

Keywords: Adipogenesis, Methylation, ABCG1, HDAC9

\footnotetext{
* Correspondence: p.froguel@imperial.ac.uk

'Department of Metabolism, Digestion and Reproduction, Imperial College

London, London, UK

Université de Lille, Inserm UMR1283, CNRS-UMR 8199 - EGID, Lille, Lille

University Hospital, F-59000 Lille, France
}

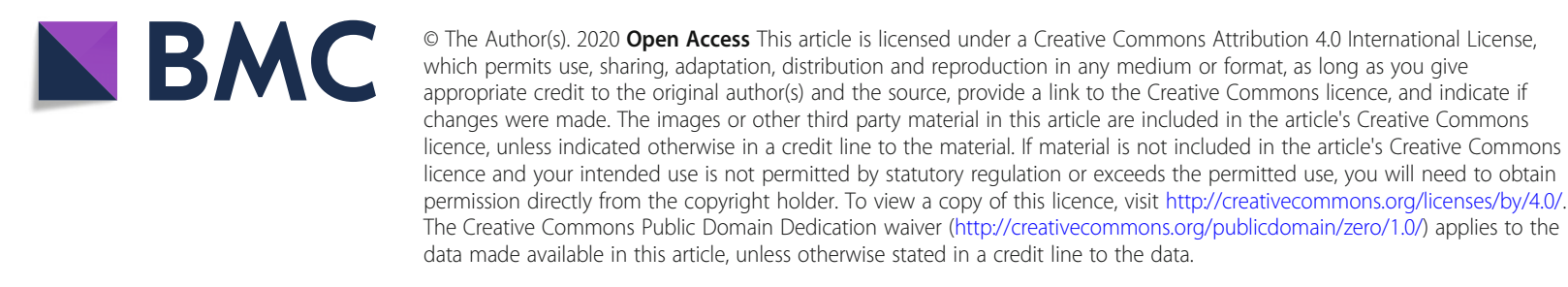




\section{Background}

Adipose tissue plays a crucial role in regulating insulin sensitivity and glucose homeostasis [1]. In obesity, adipose expansion occurs as a result of cellular hypertrophy, i.e. the increase in size of the adipocyte and/or de novo adipogenesis, which is the production of new mature adipocytes from residing preadipocytes [2-4]. Dysregulation in the adipogenic process is associated with metabolic diseases and insulin resistance [5] and is an independent risk factor for type 2 diabetes (T2D) [6]. In contrast, appropriate adipocyte expansion is protective against T2D in the context of obesity [6, 7]. Adipogenesis occurs as a result of metabolic cues that trigger the induction of key differentiation regulators, such as ADIPOQ, FASN, PPARg, ABCG1 and GLUT4 [8-11]. Epigenome-wide association studies (EWAS) have found that hypermethylation within one of these genes involved in adipogenesis, $A B C G 1$, was associated with increased body mass index (BMI), insulin resistance and T2D risk [12-15], opening avenues in the elucidation of the links between adipogenesis and metabolic diseases.

One of the most common drugs known to modulate adipogenesis are statins [16]. As a role for statins as a DNA methylation inhibitor has previously been reported [17], we hypothesised that statin treatment modulates adipogenesis by modifying the adipocyte epigenome. In this study, we confirmed the inhibitory effects of statin treatment in human preadipocytes and investigated the methylome to identify potential regulators that may be involved in adipogenesis.

\section{Results}

\section{Statin treatment reduced adipogenesis and insulin} signalling

The Simpson-Golabi-Behmel syndrome (SGBS) human preadipocyte cell line was used in this study as an in vitro model for adipocyte differentiation. In SGBS cells, lipid droplet formation occurred by 12-14 days of differentiation together with an increase in the expression of key adipogenic markers [18]. We retrieved adequate SGBS cell morphology modification and formation of lipid droplets by day 12 (Additional File 1: Figure S1a), and observed that the expression of key genes involved in adipocyte differentiation and maturation was accordingly upregulated (Additional File 1: Figure S1b).

For statin treatment, SGBS cells were differentiated for 6 days and then treated with atorvastatin and mevastatin for an additional 6 days until final maturation (Fig. 1a). We found a decrease in lipid in statin-treated SGBS cells (both atorvastatin and mevastatin) when compared to DMSO-vehicle controls $(p<0.05$; Fig. 1b). We also found that statin treatment induced a significant downregulation of many key genes associated with adipogenesis reported above (ABCG1, LEPTIN and GLUT4), with the particular exclusion of PPARG, a gene known to play a role only in the early stages of adipocytes differentiation (Fig. 1c). In adipocytes, insulin binds to the insulin receptor, which then activates a signalling cascade through the phosphorylation of Akt and Erk, thereby stimulating the translocation of GLUT4 to the plasma membrane. Therefore, analysing pAkt and pErk is a measure of insulin signalling and sensitivity. We found a decreased efficiency of insulin to activate ERK and AKT (Fig. 1d). Taken together, the data support the inhibitory effects of statin in the human adipocyte differentiation and insulin signalling, a similar effect reported in statintreated 3T3-L1 mouse adipocyte cells [16].

\section{Methylome analysis of statin-treated SGBS cell line}

To identify potential regulators involved in statin-induced adipocyte dysregulation, we performed an unbiased methylation analysis in statin-treated SGBS cells using Illumina's Infinium '850K' Methylation EPIC arrays (Additional File 1: Figure S2). We filtered differentially methylated positions (DMPs) located in the promoter region, annotated as TSS200 or TSS1500 (within 200-1500 bp from the transcription start site), in order to identify DMPs that were likely to have a biological effect. This filtering produced a total of 173,172 CpGs.

We focused our analysis on the "shared" CpGs between the two statin groups, (i.e. reproducible) and found 87 DMPs shared between the mevastatin and atorvastatin treatments (Additional File 2: Table S1, Figure S3). In mevastatin-treated cells, the most significant DMP was cg14566882, located in the promoter of the histone deacetylase $(H D A C 9)$ gene $\left(\beta=8.28 \% ; p=5.55 \times 10^{-6}\right)$ (Fig. $2 a)$. Indeed, this DMP was also significantly hypomethylated in response to atorvastatin treatment, compared to DMSO-vehicle controls $\left(\beta=5.53 \% ; p=1.35 \times 10^{-3}\right.$ ) (Fig. 2a, b). Importantly, as HDAC9 has previously been found to be involved in adipocyte differentiation [19], we focused on this gene for further biological validation.

A significant differentially methylated region (DMR) overlapping this promoter region was also found in response to both treatments (Additional File 1: Figure S4; False discovery rate $<0.05$ ). In order to validate the effect of cg14566882 hypomethylation on the expression of the HDAC9 gene, we performed qPCRs in statintreated SGBS cell lines and found significant upregulation of the HDAC9 gene at the mRNA level $(p<0.05$; atorvastatin 14-fold; mevastatin11-fold) (Fig. 2c).

ABCG1 has been reported to be regulated by HDAC9mediated changes in acetylation $[20,21]$ and may be targeted by the HDAC9 epigenetic alteration. We confirmed that ABCG1 protein expression is indeed downregulated in response to mevastatin and atorvastatin treatment (Fig. 2d). We confirmed that in response to statin, this effect was independent of the previously reported hypermethylation in $\operatorname{cg} 06500161(p$ value $>0.5)$ and cg27243685 $(p$ value $>0.5)$ 


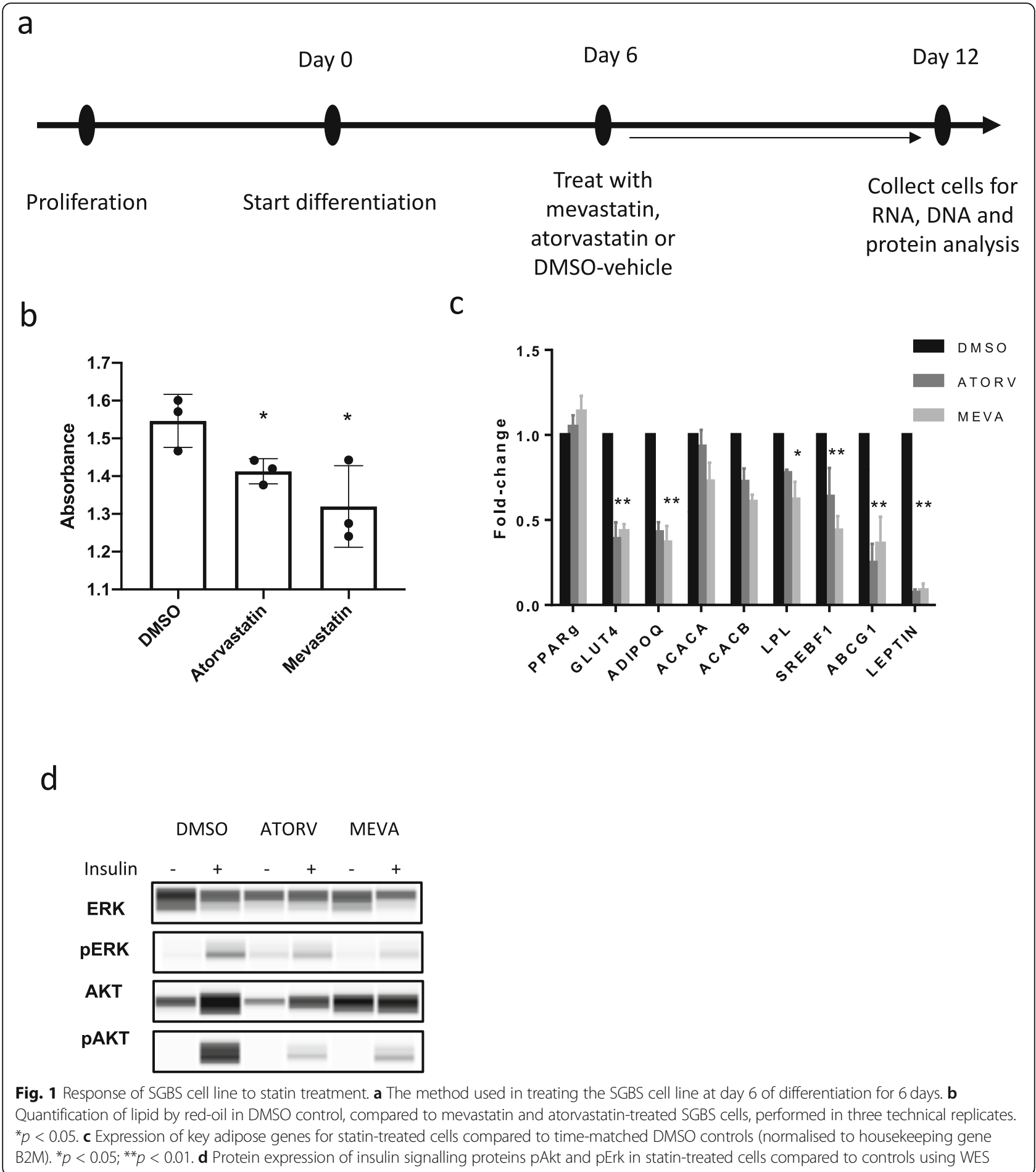

found to be associated with increased BMI and T2D incidence (Additional File 1: Table S2) [13, 14, 22, 23]. In addition, we confirmed that HDAC9 binds to the ABCG1 promoter, and this binding is increased in response to statin-treatment (Fig. 2e). This clearly illustrates that HDAC9 acts as a transcriptional repressor to ABCG1 expression in adipocytes in response to statin.

\section{Knockdown of $A B C G 1$ in SGBS preadipocytes reduced} adipocyte differentiation

We performed an $A B C G 1$ knockdown (KD) in SGBS cells to address whether reduced $A B C G 1$ expression mimics the effect of statins in adipogenesis. SGBS preadipocytes were stably transfected with a shRNA targeting ABCG1 mRNA and followed them quantitatively through 


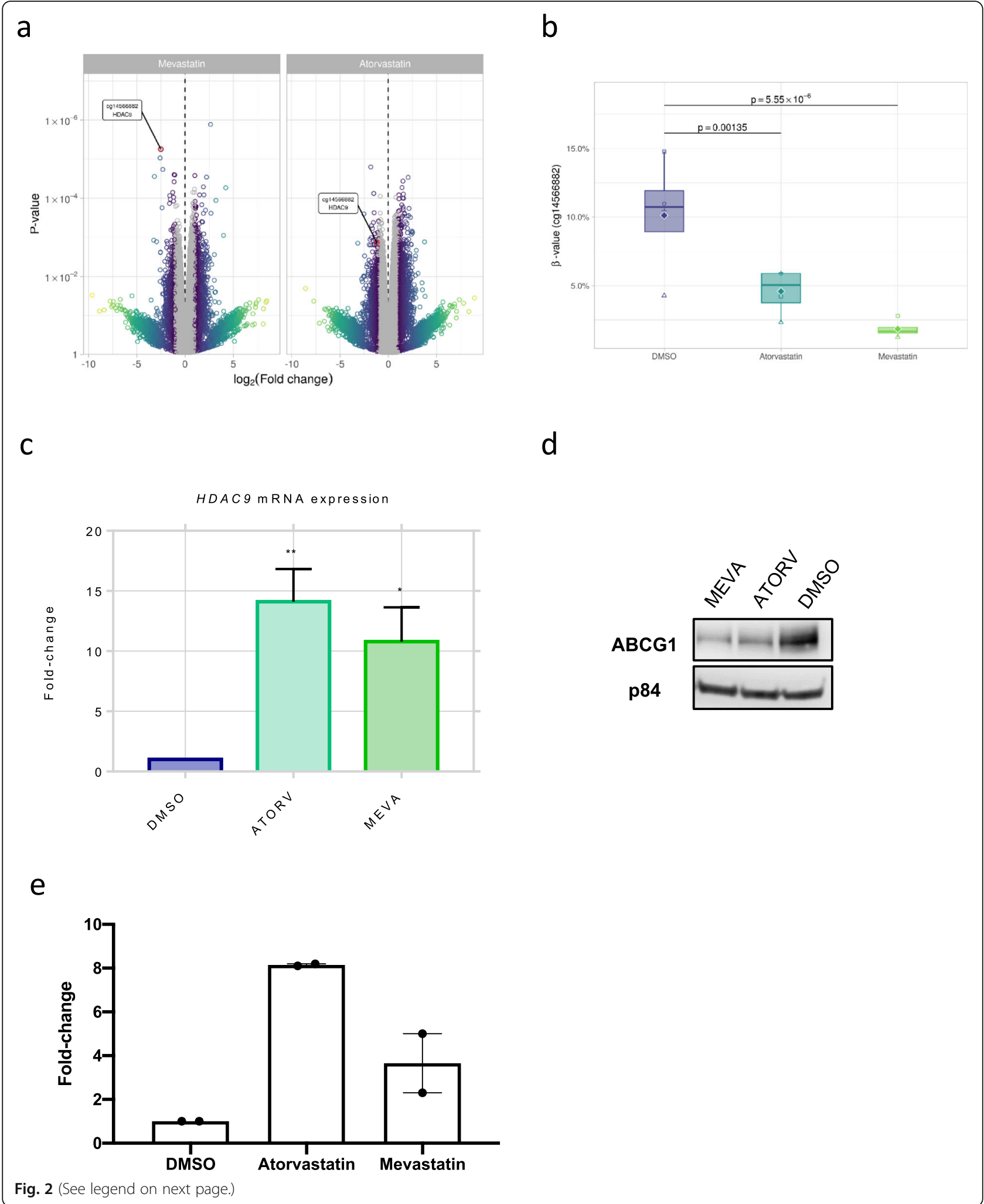


(See figure on previous page.)

Fig. 2 Methylome analysis of statin-treated SGBS cells. a Volcano plots of methylome results for statin-treated cells (grey indicates log2 fold change < 1). $\mathbf{b}$ The hypomethylation of the $\mathrm{cg} 14566882 \mathrm{CpG}$ within the HDAC9 gene in atorvastatin and mevastatin-treated cells compared to vehicle-treated DMSO cells in the 4 biological replicates (raw $\beta$-values shown). $\mathbf{c}$ The mRNA expression level of HDAC9 in mevastatin and atorvastatin-treated SGSB cell line. ${ }^{*} p<0.05$; ${ }^{* *} p<0.01$. $\mathbf{d}$ The protein expression of ABCG1 compared to housekeeping gene $\mathrm{p} 84$ shows a reduced expression in atorvastatin and mevastatin-treated SGBS cells. e ChIP qPCR using anti-HDAC9 antibody for the ABCG1 promoter for control (DMSO), mevastatin and atorvastatintreated cells, normaled to no antibody (mock lgG) control. This experiment was performed in two biological replicates

maturation and differentiation, and this data was compared with cells transfected with a non-targeting shRNA (control). A similar protocol for stably knockdown Abcg1 via shRNA has previously been achieved and described in mouse 3T3-L1 preadipocytes [24]. We initially analysed the expression of ABCG1 protein in normal adipocytes to show that it is positively associated with adipogenesis as ABCG1 starts to become expressed at day 6 of differentiation (Fig. 3a). The efficient silencing of ABCG1 (ABCG1 $\mathrm{KD})$ was confirmed at the protein level (Fig. 3b). ABCG1 KD in SGBS cells was accompanied by a significant reduction in the lipid content ( $20 \%$ reduction, $p<0.05$; Fig. $3 c$ ), along with the downregulation of the following adipocyte differentiation markers FASN, PPARG and PLIN1 and key adipocyte maturation markers ADIPOQ and GLUT4 (Fig. $3 \mathrm{~d})$. As a consequence of impaired adipogenesis, the $A B C G 1 \mathrm{KD}$ led to a significant reduction in glucose uptake stimulated by insulin (65\% reduction, $p<0.001$; Fig. $3 e)$. In addition, we found a decreased efficiency of insulin to activate AKT in those cells (Fig. 3f). As a whole, this data indicates that $A B C G 1$ levels are pivotal for the control of human adipocyte differentiation and glucose metabolism.

\section{$A B C G 1$ is downregulated in response to statin in human blood samples}

We next explored whether $A B C G 1$ was also dysregulated in samples from human subjects. We analysed reported transcriptomic data from blood samples from two cohorts. The first consisted of a total of 57 individuals from the ECLIPSE cohort, of which 13 were statin users [25]. A significant reduction in the expression of $A B C G 1$ in the statin group $\left(p=1.41 \times 10^{-5}\right)$ was found, compared to nonusers (Fig. 4a). In addition, we also analysed data from the YELLOW II study [26], a retrospective study following 85 individuals before and following an extensive 8-12 week statin therapy. In peripheral blood mononuclear cells obtained from blood samples, $A B C G 1$ expression was significantly decreased following statin treatment, compared to baseline levels, for two ABCG1 probes (ILMN_1794782 $p$ $=2.76 \times 10^{-5}$; ILMN_2329927 $p=4.28 \times 10^{-4}$ ) (Fig. 4b). Taken together, this demonstrates that $A B C G 1$ reduction in response to statin is indeed reflected in human blood samples. Of note, no data on HDAC9 expression was available in the ECLIPSE case control study, and no significant change in $H D A C 9$ expression was reported in the intervention YELLOW II study, maybe due to the lack of sufficient statistical power of this study.

\section{Discussion}

A recent 15-year prospective study found a staggering $38 \%$ increased incidence of $\mathrm{T} 2 \mathrm{D}$ in statin users, regardless of the type of statin used [27]. Here, we report that two statins, atorvastatin and mevastatin, hamper the differentiation process in the SGBS human preadipocyte cell line and decreased insulin sensitivity.

We focused our analysis on promoter DMPs, which are normally inversely correlated with expression [28, 29]. Therefore, not surprisingly, given the inhibitory effect of statin, our $850 \mathrm{~K}$ methylation analysis revealed that most DMPs were hypermethylated. This includes the IDI1 gene, which encodes the isopentenyl diphosphate isomerase, a component of the cholesterol synthesising pathway [30, 31].

We report for the first time that statin treatment was associated with a significant hypomethylation of HDAC9 promoter, which is inversely correlated with $H D A C 9$ gene expression. These findings are of particular significance in light of several studies that demonstrated the key role of HDAC9 in adipocytes function: overexpression of Hdac9 in 3T3-L1 preadipocyte mouse cell lines suppressed adipogenesis, and inversely, preadipocytes isolated from Hdac 9 knockout mice had an accelerated adipocyte differentiation [32]. Furthermore, Hdac9 knockout mice showed improved metabolic homeostasis and were protected from adipose tissue dysfunction in mice fed on a high-fat feeding [19]. These studies clearly indicate the deleterious role of HDAC9 in maintaining adipocyte homeostasis both in vitro and in vivo.

In a study, it was shown that HDAC9-deficient macrophages and monocytes were directly involved in $A B C G 1$ transcription $[20,21]$. Indeed, we confirmed in our adipocyte in vitro model that $H D A C 9$ binds directly to the $A B C G 1$ promoter and acts as a transcriptional repressor for $A B C G 1$ expression. This is of particular interest, as several studies have reported a role of ABCG1 in obesity, insulin resistance and T2D. Elevated ABCG1 expression is associated with increased fat mass from obese individuals, suggesting that $A B C G 1$ is also involved in human adipogenesis [24]. Although genome-wide association studies have not found any single nucleotide polymorphisms (SNPs) within or nearby $A B C G 1$ associated with 
a

C

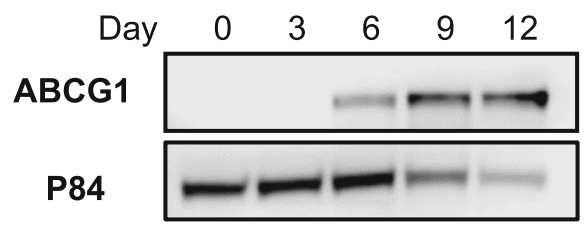

Lipid content in ABCG1 KD day 12

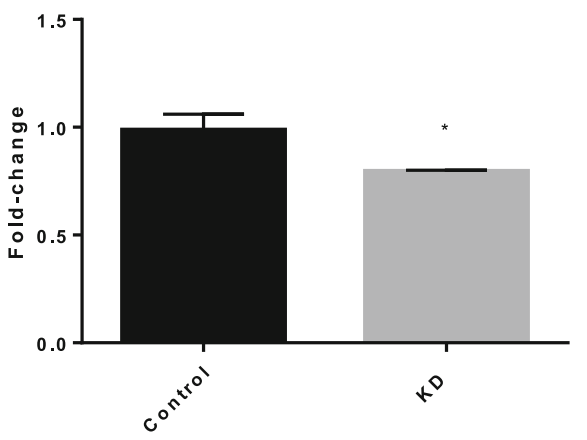

e

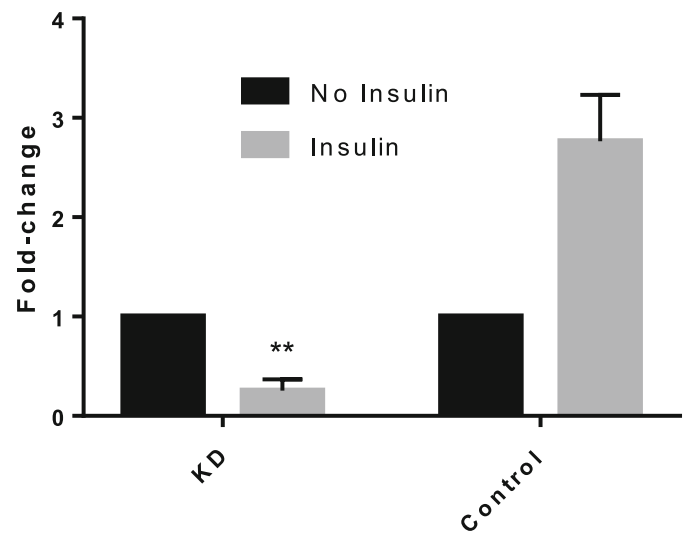

b

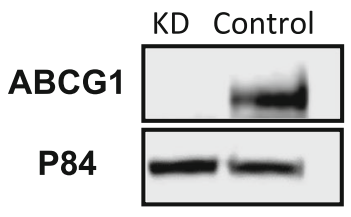

d

$A B C G 1 K D$ in adipocytes

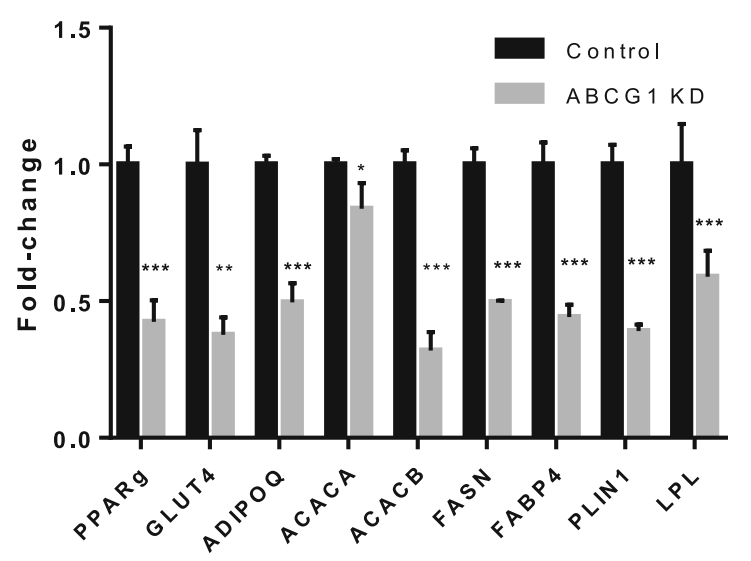

$f$ 

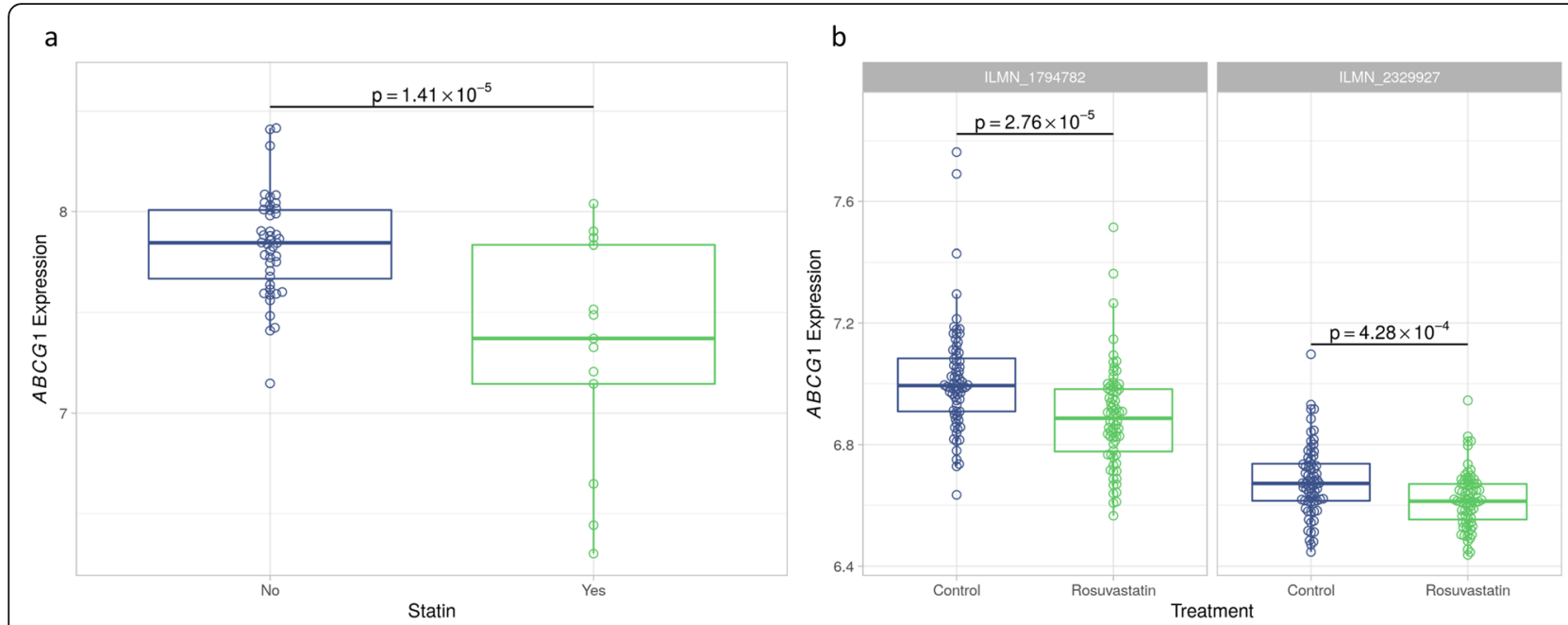

Fig. 4 The expression of $A B C G 1$ in human samples. a $A B C G 1$ expression was reduced in 13 statin-treated individuals compared to control nonusers using transcriptomic data. b Data from a total of 85 samples, after extensive statin treatment for $8-12$ weeks, there was a reduction in ABCG1 expression compared to baseline levels in two probes

increased T2D risk, several EWAS have found that hypermethylation in the $A B C G 1$ gene was associated with fasting glucose, HbA1C levels, lipid metabolism, fasting insulin, T2D risk and BMI [13-15, 33-36]. Additional observations in mouse models have shown that $\mathrm{Abcg} 1^{-1-}$ mice were protected from high-fat diet-induced glucose intolerance [37]. A recent study found that ABCG1 expression is reduced in both subcutaneous and visceral adipose tissue in morbidly obese patients with metabolic syndrome compared to those without metabolic syndrome, providing further evidence for a role of $A B C G 1$ in the maintenance of metabolic homeostasis in adipocytes [38]. In addition, two studies showed that $A B C G 1$ expression was decreased in blood white human cells in response to statins. As ABCG1 was downregulated in response to statin, we hypothesised that ABCG1 plays a role in statin-induced adipocyte dysregulation.

Indeed, we showed that $A B C G 1$ expression increases during human SGBS adipocyte differentiation and through $A B C G 1$ silencing, confirm that the level of $A B C G 1$ expression is crucial for the appropriate expression of lipid metabolism markers, which include FASN, FABP4, PLIN1 and PPARG, for correct human adipocyte differentiation. Our findings are consistent with previous data showing variation in these four genes following Abcg1 silencing in mouse 3T3-L1 pre-adipocyte cells [24]. The downregulation of GLUT4 in ABCG1 KD suggested a decrease in insulin-induced glucose uptake. Indeed, we confirmed a downregulation of phosphorylation of AKT and ERK. Collectively, this data indicates that normal ABCG1 function is required for adipogenesis and insulin signalling. In addition, we have confirmed using two separate datasets that statin use was indeed correlated with a reduction in
ABCG1 expression in human blood samples. Other studies have reported a link between ABCG1 downregulation and diabetes incidence [39] and high fasting glycaemia [40]. Taken together, our human cellular data is consistent with human observational studies, in which the inhibition of $A B C G 1$ expression was deleterious for metabolism in adipose tissue.

Our results focused on the effects of cg14566882 hypomethylation on HDAC9 expression; however, it is important to note that these changes could be mediated by other indirect $\mathrm{CpG}$ changes. Furthermore, despite the fact that we show that HDAC9 directly binds to the $A B C G 1$ promoter as a statin effect, the relationship between cg14566882 hypomethylation and HDAC9 expression is correlative.

\section{Conclusions}

The model proposed based on our data from statininduced insulin resistance is that hypomethylation of the $H D A C 9$ promoter is correlated with $H D A C$ gene expression, which acts as a transcriptional repressor to $A B C G 1$ expression and thereby adipocyte differentiation and metabolic dysfunction (Fig. 5). Adipocyte turnover by adipogenesis is crucial for the maintenance of metabolic homeostasis and insulin sensitivity [41]. Our data provides a novel epigenetic link between adipogenesis dysfunction and insulin resistance, mediated by statins. The increased understanding of adipogenesis provides a promising new avenue for the treatment of metabolic disease in obesity $[8,41]$. Both HDAC9 and ABCG1 have been proposed as therapeutic targets for patients with obesity in separate previous studies [19, 24]; however, our data support a mechanistic pathway linking them to metabolic diseases. 


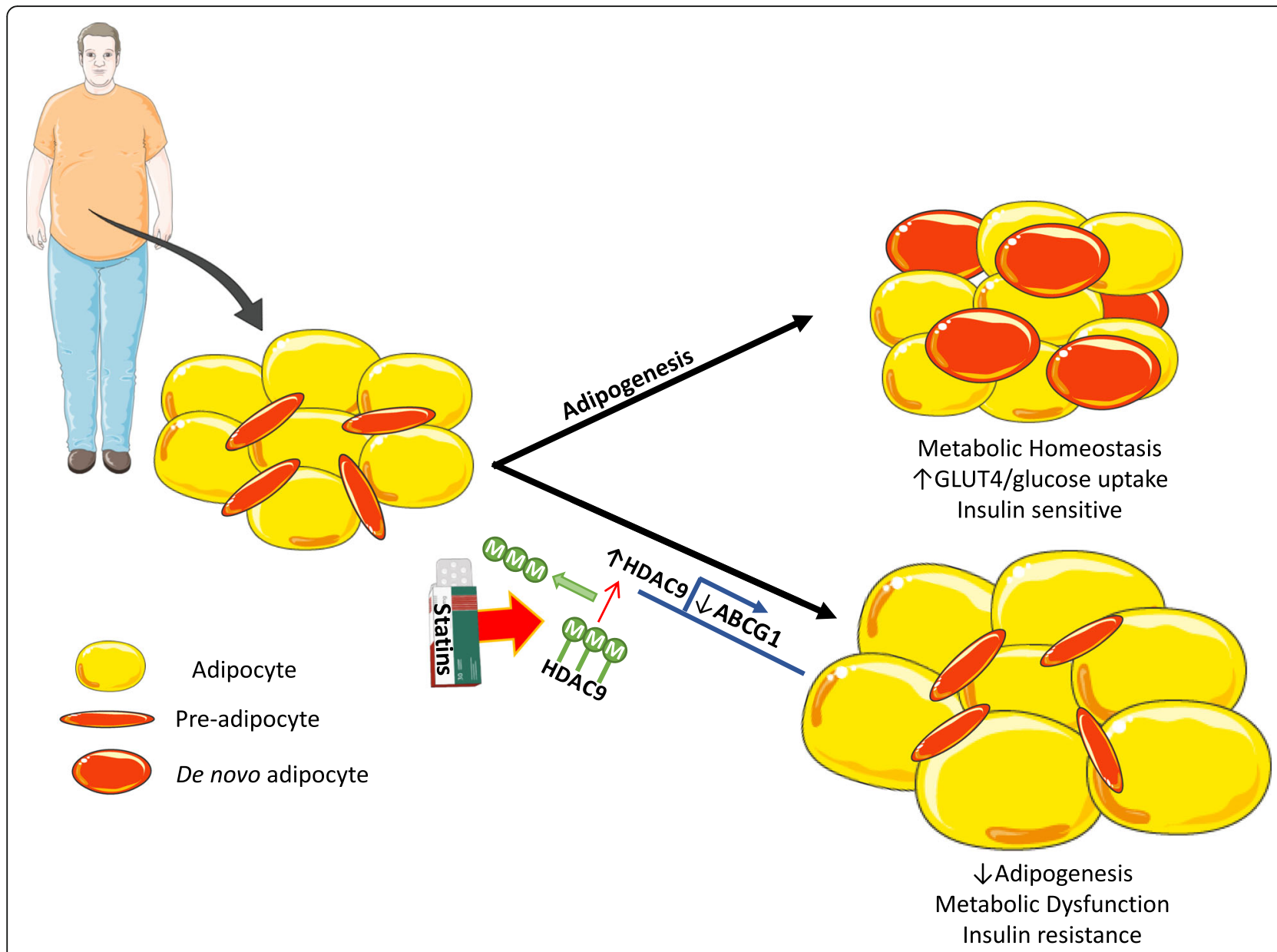

Fig. 5 A schematic representation of the role of adipocyte turnover in health and disease. In healthy individuals, preadipocytes differentiate into mature adipocytes, which have a role in maintaining insulin sensitivity. However, in response to statins, epigenetic changes in HDAC9 cause acetylation changes in $A B C G 1$ and other crucial adipogenesis genes, which in-turn lead to an obstruction of differentiation and metabolic dysfunction

\section{Methods}

\section{Cell culture and differentiation of SGBS cell line}

For all the in vitro experiments, we used the SGBS cell line, a pre-adipocyte cell line that has not been transformed or immortalised and has the capacity to retain adipocyte differentiation in up to 50 passages [18]. This cell line was kindly provided by Prof. Dr. M. Wabitsch (University of Ulm, Germany) and maintained in DMEM/F12 supplemented with $10 \%$ foetal bovine serum and $0.01 \%$ penicillin/ streptomycin (15140-122 - Life Technologies), as previously described [18]. Confluent preadipocytes were differentiated under serum-free culture conditions by washing twice with phosphate-buffered saline (PBS) and then exposing to DMEM/F12 supplemented with $2 \mu \mathrm{mol} / \mathrm{l}$ rosiglitazone, 25 $\mathrm{nmol} / \mathrm{l}$ dexamethasone, $0.5 \mathrm{mmol} / \mathrm{l}$ methylisobuthylxantine, $0.1 \mu \mathrm{mol} / \mathrm{l}$ cortisol, $0.01 \mathrm{mg} / \mathrm{ml}$ transferrin, $0.2 \mathrm{nmol} / \mathrm{l}$ triiodotyronin and $20 \mathrm{nmol} / \mathrm{l}$ human insulin for 4 days. The cells were then cultured for a further 8 days in fresh DMEM/F12 supplemented with $0.1 \mu \mathrm{mol} / \mathrm{l}$ cortisol, 0.01 $\mathrm{mg} / \mathrm{ml}$ transferrin, $0.2 \mathrm{nmol} / \mathrm{l}$ triiodotyronin and $20 \mathrm{nmol} / \mathrm{l}$ human insulin. Microscopic images of SGBS cells were taken under a microscope (IT404; VWR) using the Motic Image plus version 2.0 (Motic Europe).

\section{Treatment with statins}

At 6 days of differentiation, SGBS cells were treated with $10 \mu \mathrm{M}$ mevastatin (M2537, Sigma Aldrich) or atorvastatin (PZ0001, Sigma Aldrich) and compared to a dimethyl sulfoxide (DMSO)-vehicle control (D2650, Sigma Aldrich). The cells were then incubated for a further 6 days. On day 12 of differentiation, cells were collected for further analysis.

\section{K Illumina methylation analysis}

DNA was extracted from SGBS cells treated with atorvastatin and mevastatin at day 6 for 6 days and collected the cells at day 12. DNA was extracted using the NucleoSpin Tissue kit (Takara Bio). Bisulfite conversion of $500 \mathrm{ng}$ genomic DNA was performed using the EZ-96 
DNA Methylation kit (Zymo Research) following the manufacturer's protocol. Bisulfite-converted DNA was subjected to genome-wide DNA methylation analysis using Illumina's Infinium '850K' Methylation EPIC array to identify differentially methylated positions (DMPs). The $850 \mathrm{~K}$ methylationEPIC array was carefully designed to include a total of 850,000 CpGs from ENCODE open chromatin and enhancer, DNase hypersensitive sites and CpG islands. The resulting DNA methylation IDAT files were imported using the minfi $\mathrm{R}$ package for further processing and quality control [42]. The following CpG probes were excluded from further analysis: probes on sex chromosomes, cross-hybridising probes, non-cg probes and probes that lie near single nucleotide polymorphisms (SNPs). Probe-design biases and batch effects were normalised using R packages ENmix [43] and SVA (ComBat) [44], respectively.

To identify DMPs, the $\mathrm{R}$ package limma was used [45]. The model included treatment (atorvastatin, mevastatin or DMSO-vehicle) as a categorical variable and replicates/day of experiment as a covariate. Methylation levels denoted by beta-values, where 0 indicates $0 \%$ methylation and 1 indicates 100\% methylation, were transformed to $M$ values [46]. All statistical significance is based on the nominal $p$ values. To identify differentially methylated regions (DMRs), the $\mathrm{R}$ package DMRcate was used [47], which uses Gaussian kernel smoothing based on the DMPs. All DMRs throughout the genome were tested.

\section{RNA extraction, CDNA conversion and RT-PCR}

Total RNA was extracted from cultured cells using a RiboPure RNA Purification Kit (AM1924; Invitrogen), according to the manufacturer's instructions and quantified on a NanoDrop Spectrophotometer (Thermo Scientific). RNA was reverse transcribed using a High-Capacity RNA-tocDNA Kit (4387406; Applied Biosystems), according to the manufacturer's instructions. qPCRs were conducted on an Applied Biosystems 7900HT Fast Real-Time PCR System, and quantitative expression levels were obtained using the SDS v2.3 Software (Applied Biosystems) using Taqman Gene Expression Assays (ThermoFisher Scientific). The following probes were used: ABCG1 (Hs01555193_m1), CEBPB (Hs00270923_s1), LPL (Hs00173425_m1), ACACA (Hs01046047_m1) FABP4 (Hs01086177_m1), GLUT4 (Hs00168966_m1), ACACB (Hs00153715_m1), PPARG (Hs01115513_m1), CEBPA (Hs00269972_s1), ADIPOQ (Hs00605917_m1), FASN (Hs01005622_m1), SREBF1 (Hs01088691_m1) and PLIN1 (Hs00160173_m1) (Life Technologies). Each reaction was normalised to a beta-2microglobulin (B2M) control (Hs00984230_m1; Applied Biosystems). For quantifications using SYBR-green, qPCRs were performed using (SsoAdvanced Universal SYBR Green Supermix, BioRad) using the BioRad CFX96 Real-
Time PCR Detection System (Biorad). HDAC9 gene primer sequences were forward: AGTGGCAGAGAGGAGAAG CA and reverse: CAGTTCTCCAGGCTCTGGTC. At least three biological replicates were performed, and the data represents the means \pm SEM. A two-tailed $t$ test was performed using GraphPad Prism (GraphPad software Inc., La Jolla, USA), and $p$ value $<0.05$ was considered to be statistically significant.

\section{Lipid quantification}

Culture medium was removed, and the cells were washed twice with PBS and then fixed with 4\% PFA for $10 \mathrm{~min}$. Fixed cells were washed twice with water and incubated in $60 \%$ isopropanol twice. The alcohol was discarded, and the cells were then incubated in an Oil Red O (Sigma) and water solution (3:2) for $5 \mathrm{~min}$. Cells were rinsed three times with water, and then $100 \%$ isopropanol was added to extract the red oil. The absorbance was measured at $5490 \mathrm{~nm}$ on a microplate reader.

\section{Western blotting}

Cells were washed twice in ice-cold PBS and harvested in RIPA buffer (ThermoFisher Scientific) supplemented with protease inhibitors. Cell lysates were centrifuged at $20,000 \times g$ for $20 \mathrm{~min}$ at $4{ }^{\circ} \mathrm{C}$, and the total protein lysate was quantified using Bradford Reagent (B6916; Sigma). Then, $40 \mu \mathrm{g}$ of total protein lysate was separated on a 4$12 \%$ Bis-Tris Plus Gel (Life Technologies) and transferred to a nitrocellulose membrane using the iBlot2 Gel Transfer Device (Life Technologies). Membranes were blocked in $0.5 \%$ non-fat dry milk and probed with antiABCG1 (1:1000; ab52617, abcam) and antinuclear matrix protein $\mathrm{p} 84$ (1:5000; ab487, abcam) primary antibodies anti-rabbit IgG (1:20,000; Ab205718, abcam), and anti-mouse IgG (1:5000; A4416, Sigma Aldrich) secondary antibodies for $1 \mathrm{~h}$ at room temperature. Membranes were exposed using Clarity Western ECL Substrate (BioRad), and protein bands were detected on a LI-COR Imaging system with the C-DiGit Image Studio 4.0 software (LI-COR Biosciences, Ltd., UK).

\section{Phosphorylated AKT analysis}

Differentiated cells cultured in DMEM/F12 medium were serum starved overnight and then washed with PBS and stimulated with or without $200 \mathrm{nM}$ insulin for $1 \mathrm{~h}$ in DMEM/F12 (without glucose or serum) at $37^{\circ} \mathrm{C}$ in $5 \%$ $\mathrm{CO}_{2}$. The total protein was harvested in RIPA buffer supplemented with protease and phosphatase inhibitors, as described above. The primary antibodies (all used at 1: 1000 dilution unless otherwise stated) used were anti pAKT (S473; Cell Signaling) and anti Akt (9272 - 1:5,000 dilution; Cell Signaling), and the secondary antibody used was goat pAb to Rb igG (Ab205718 - 1:20,000 dilution; Abcam). Protein expression studies were also performed 
by WES, an automated capillary-based size separation and nano-immunoassay system (ProteinSimple, San Jose CA, USA - a Bio-Techne Brand), according to manufacturer's protocol, for analysis performed using the Compass for Simple Western software v.4.0. The WES was performed on SGBS samples (1:100) for anti pAKT (S473; Cell Signaling), anti Akt (9272 - 1:100 dilution; Cell Signaling), anti pERK (9102; Cell Signaling) and anti ERK (9101 - 1: 100 dilution; Cell Signaling).

\section{Glucose uptake assay}

A Glucose Uptake-Glo Assay (J1342; Promega) was used to measure glucose uptake in differentiated cells (day 12 ), according to the manufacturer's protocol. A total of 20,000 cells in $100 \mu \mathrm{l}$ media were plated in each well of a 96-well white plate. Differentiated cells were cultured overnight in DMEM/F12 media with no serum. On the day of the assay, the media was replaced with $100 \mu \mathrm{l}$ DMEM/F12 (without glucose or serum) supplemented with or without $1 \mu \mathrm{M}$ insulin and incubated for $1 \mathrm{~h}$ at $37^{\circ} \mathrm{C}$ in $5 \% \mathrm{CO}_{2}$. Cells were washed with PBS and $50 \mu \mathrm{l}$ $1 \mathrm{mM}$ 2-Deoxy-D-glucose (2DG) was added to each well and incubated for $10 \mathrm{~min}$ at room temperature. Next, $25 \mu \mathrm{l}$ Stop Buffer was added followed by $25 \mu \mathrm{l}$ Neutralization Buffer per well. Finally, $100 \mu$ 2 DG6P Detection Reagent was added and incubated for $4.5 \mathrm{~h}$ at room temperature. The luminescence was recorded on a Mithras LB 940 luminometer (Berthold Technologies) and analysed using the MicroWin Software (Berthold Technologies).

\section{Chromatin immunoprecipitation}

Following differentiation and statin treatment, chromatin immunoprecipitation was performed using a ChIP assay (Abcam, ab500). A total of $3 \times 10^{6}$ cells per condition (DMSO, mevastatin and atorvastatin-treatment) were sheared to 200-700 bp using the Covaris AFATM (Adaptive Focused Acoustics) technology (200 cycles per burst and $8 \mathrm{~min}$ processing time). The sheared cells were incubated overnight with anti-HDAC9 antibody (ab59718) or negative control (no antibody). DNA-purified samples were analysed using SYBR green RT-PCR to amplify the $A B C G 1$ promoter region using the forward primer $5^{\prime}$ GAGCCTCGCCAGCTCATTAT 3' and the reverse primer 5' TCCCAAGTGTTGCCCAAAGA 3'.

\section{$A B C G 1$ silencing using shRNA lentiviral vector}

Undifferentiated SGBS cells were plated at 50\% confluence in 6-well plates and infected with commercial lentiviral particles targeting either human ABCG1 (TRCN0000420907; Sigma) (TAGGAAGATGTAGGCAGATTG) or non-target controls (SHC202) (CCGGCAACAAGATGAAGAGCAC CAACTC) and (TRCN0000158395; CCTACAGTGGATG TCCTACAT) (Sigma Aldrich). The transduced cells were selected in media containing $1 \mu \mathrm{g} / \mathrm{ml}$ puromycin for 6 days. Stable $A B C G 1 \mathrm{KD}$ and control cells were then cultured and differentiated into mature adipocytes, as described above.

\section{Analysis of transcriptomic data from human samples} Transcriptomic data (Affymetrix Human Gene 1.1 ST Array) from the GSE71220 dataset was downloaded from the GEO database. The subjects analysed were the 57 control samples from the Evaluation of COPD Longitudinally to Identify Predictive Surrogate Endpoints (ECLIPSE) study, of which 13 were statin-users [25]. In addition, transcriptomic data (Illumina HumanHT-12 WG-DASL V4.0 R2 expression beadchip) from peripheral blood mononuclear cells from the YELLOW II retrospective study (GSE86216) was also downloaded from the GEO dataset. This included blood samples from a total of 85 patients that were analysed before and after an extensive 8-12 week statin treatment [26]. For both datasets, the data was downloaded and analysed using the R packages GEOquery [42] and limma [45].

\section{Supplementary information}

Supplementary information accompanies this paper at https://doi.org/10. 1186/s13148-020-00858-w.

\begin{abstract}
Additional file 1 - Supplementary Figures. Fig. S1: Differentiation of SGBS cell line. (a) Micorscopic mages of lipid droplets in pre-adipocytes (day 0 ) and differentiated mature adipocytes (day 12) visualised using microscopy (x10 magnification - scale bar $10 \mu \mathrm{M})$ (b) Expression of key adipose genes during a 12-day differentiation period (normalised to housekeeping gene B2M). For each gene, the fold-change compared to the day expression is first observed is presented. Data represent three biological replicates and plotted as the mean \pm SEM. Fig. S2: All DMPs identified in response to a) mevastatin and b) atorvastatin treatment. Fig. S3: DMPs annotated to the promoter region that were also shared between mevastatin and atorvastatin treatments. Grey indicates $\log 2$ fold change < 1. Fig. S4: Differentially methylated region (DMR) in HDAC9 promoter shared between mevastatin and atorvastatin treatments.

Additional file $\mathbf{2}$ - Supplementary Tables. Table S1: Details of shared DMPs between mevastatin and atorvastatin-treated SGBS cells. Table S2: Details of CpG results in SGBS statin-treated cells in already reported to be associated wih BMI and T2D incidence.
\end{abstract}

\section{Abbreviations \\ B2M: Beta-2-microglobulin; BMI: Body mass index; DMP: Differentially methylated position; DMR: Differentially methylated region; DMSO: Dimethyl sulfoxide; EWAS: Epigenome-wide association study; HDAC9: Histone deacetylase 9; KD: Knockdown; SGBS: Simpson-Golabi-Behmel syndrome; SNP: Single nucleotide polymorphism; T2D: Type 2 diabetes}

\section{Acknowledgements}

The authors would like to thank Prof. Dr. M. Wabitsch (University of Ulm, Germany) for kindly providing the SGBS cell line and differentiation protocol.

\section{Authors' contributions}

AK, AA, TA, FT and PF designed the project. AK, FT, SM, RB and HC have performed the experiments. SL performed the methylome wet lab experiments and MC performed the methylation analysis. AK and MC prepared the figures. AK and PF wrote the manuscript. All authors edited the paper. 


\section{Funding}

This study was supported by non-profit organisations and public bodies for funding of scientific research conducted in France and within the European Union: "Centre National de la Recherche Scientifique", "Université de Lille 2", "Institut Pasteur de Lille", "Société Francophone du Diabète", "Contrat de Plan Etat-Région", "Agence Nationale de la Recherche", ANR-10-LABX-46, ANR EQUIPEX Ligan MP: ANR-10-EQPX-07-01, European Research Council GEPIDIAB 294785.

\section{Availability of data and materials}

The datasets generated and/or analysed during the current study are available in the Gene Expression Omnibus (GEO) repository, under GSE139211 https://www.ncbi.n/m.nih.gov/geo/query/acc.cgi?acc=GSE139211.

\section{Ethics approval and consent to participate}

Not applicable

\section{Consent for publication}

Not applicable

\section{Competing interests}

The authors declare that they have no competing interests.

\section{Received: 21 November 2019 Accepted: 28 April 2020}

\section{Published online: 14 May 2020}

\section{References}

1. Scherer PE. The multifaceted roles of adipose tissue - therapeutic targets for diabetes and beyond: The 2015 Banting Lecture. Diabetes. 2016 Jun 1;65(6): 1452-61.

2. Arner $E$, Westermark $P O$, Spalding $K L$, Britton T, Rydén $M$, Frisén J, et al. Adipocyte turnover: relevance to human adipose tissue morphology. Diabetes. 2010 Jan:59(1):105-9.

3. Cotillard A, Poitou C, Torcivia A, Bouillot J-L, Dietrich A, Klöting N, et al. Adipocyte size threshold matters: link with risk of type 2 diabetes and improved insulin resistance after gastric bypass. J Clin Endocrinol Metab. 2014 Aug;99(8):E1466-70.

4. Muir LA, Baker NA, Washabaugh AR, Neeley CK, Flesher CG, DelProposto JB, et al. Adipocyte hypertrophy-hyperplasia balance contributes to weight loss after bariatric surgery. Adipocyte. 2017 03:6(2):134-140.

5. Gustafson B, Hedjazifar S, Gogg S, Hammarstedt A, Smith U. Insulin resistance and impaired adipogenesis. Trends Endocrinol Metab TEM. 2015; 26(4):193-200

6. Lönn M, Mehlig K, Bengtsson C, Lissner L. Adipocyte size predicts incidence of type 2 diabetes in women. FASEB J Off Publ Fed Am Soc Exp Biol. 2010; 24(1):326-31.

7. Weyer C, Foley JE, Bogardus C, Tataranni PA, Pratley RE. Enlarged subcutaneous abdominal adipocyte size, but not obesity itself, predicts type II diabetes independent of insulin resistance. Diabetologia. 2000;43(12): 1498-506.

8. Ghaben AL, Scherer PE. Adipogenesis and metabolic health. Nat Rev Mol Cell Biol. 2019;20(4):242-58.

9. Hu E, Liang P, Spiegelman BM. AdipoQ is a novel adipose-specific gene dysregulated in obesity. J Biol Chem. 1996;271(18):10697-703.

10. Hu E, Tontonoz P, Spiegelman BM. Transdifferentiation of myoblasts by the adipogenic transcription factors PPAR gamma and C/EBP alpha. Proc Natl Acad Sci U S A. 1995:92(21):9856-60.

11. Lowe CE, O'Rahilly S, Rochford JJ. Adipogenesis at a glance. J Cell Sci. 2011; 124(16):2681-6.

12. Braun KVE, Dhana $K$, de Vries PS, Voortman T, van Meurs JBJ, Uitterlinden AG, et al. Epigenome-wide association study (EWAS) on lipids: the Rotterdam Study. Clin Epigenetics. 2017;9:15

13. Chambers JC, Loh M, Lehne B, Drong A, Kriebel J, Motta V, et al. Epigenome-wide association of DNA methylation markers in peripheral blood from Indian Asians and Europeans with incident type 2 diabetes: a nested case-control study. Lancet Diabetes Endocrinol. 2015:3(7):526-34.

14. Dayeh T, Tuomi T, Almgren P, Perfilyev A, Jansson P-A, de Mello VD, et al. DNA methylation of loci within $\mathrm{ABCG} 1$ and $\mathrm{PHOSPHO} 1$ in blood DNA is associated with future type 2 diabetes risk. Epigenetics. 2016;11(7):482-8.
15. Wilson LE, Harlid S, Xu Z, Sandler DP, Taylor JA. An epigenome-wide study of body mass index and DNA methylation in blood using participants from the Sister Study cohort. Int J Obes 2005. 2017 Jan;41(1):194-9.

16. Nakata M, Nagasaka S, Kusaka I, Matsuoka H, Ishibashi S, Yada T. Effects of statins on the adipocyte maturation and expression of glucose transporter 4 (SLC2A4): implications in glycaemic control. Diabetologia. 2006;49(8):188192.

17. Kodach LL, Jacobs RJ, Voorneveld PW, Wildenberg ME, Verspaget HW, van Wezel T, et al. Statins augment the chemosensitivity of colorectal cancer cells inducing epigenetic reprogramming and reducing colorectal cancer cell 'stemness' via the bone morphogenetic protein pathway. Gut. 2011; 60(11):1544-53.

18. Wabitsch M, Brenner RE, Melzner I, Braun M, Möller P, Heinze E, et al. Characterization of a human preadipocyte cell strain with high capacity for adipose differentiation. Int J Obes Relat Metab Disord J Int Assoc Study Obes. 2001;25(1):8-15

19. Chatterjee TK, Basford JE, Yiew KH, Stepp DW, Hui DY, Weintraub NL. Role of histone deacetylase 9 in regulating adipogenic differentiation and high fat diet-induced metabolic disease. Adipocyte. 2014;3(4):333-8.

20. Cao Q, Rong S, Repa JJ, Clair RS, Parks JS, Mishra N. HDAC9 represses cholesterol efflux and generation of alternatively activated macrophages in atherosclerosis development. Arterioscler Thromb Vasc Biol. 2014;34(9):1871-9.

21. Jiang W, Agrawal DK, Boosani CS. Cell-specific histone modifications in atherosclerosis (Review). Mol Med Rep. 2018;18(2):1215-24

22. Demerath EW, Guan W, Grove ML, Aslibekyan S, Mendelson M, Zhou Y-H, et al. Epigenome-wide association study (EWAS) of BMI, BMI change and waist circumference in African American adults identifies multiple replicated loci. Hum Mol Genet. 2015;24(15):4464-79.

23. Rönn $T$, Volkov P, Gillberg L, Kokosar M, Perfilyev A, Jacobsen AL, et al. Impact of age, $\mathrm{BMI}$ and $\mathrm{HbA} 1 \mathrm{c}$ levels on the genome-wide DNA methylation and mRNA expression patterns in human adipose tissue and identification of epigenetic biomarkers in blood. Hum Mol Genet. 2015; 24(13):3792-813

24. Frisdal E, Le Lay S, Hooton $H_{1}$, Poupel L, Olivier M, Alili $\mathrm{R}$, et al. Adipocyte ATP-binding cassette G1 promotes triglyceride storage, fat mass growth and human obesity. Diabetes. 2015:64(3):840-55.

25. Obeidat M, Fishbane N, Nie Y, Chen V, Hollander Z, Tebbutt SJ, et al. The effect of statins on blood gene expression in COPD. PLOS One. 2015;10(10): e0140022.

26. Chamaria S, Johnson KW, Vengrenyuk Y, Baber U, Shameer K, Divaraniya AA et al. Intracoronary imaging, cholesterol efflux, and transcriptomics after intensive statin treatment in diabetes. Sci Rep [Internet]. 2017 Aug 1 [cited 2019 Nov 77;7. Available from: https://www.ncbi.n/m.nih.gov/pmc/articles/ PMC5539108/.

27. Ahmadizar F, Ochoa-Rosales C, Glisic M, Franco OH, Muka T, Stricker BH. Associations of statin use with glycaemic traits and incident type 2 diabetes. Br J Clin Pharmacol. 2019 May;85(5):993-1002.

28. Anastasiadi D, Esteve-Codina A, Piferrer F. Consistent inverse correlation between DNA methylation of the first intron and gene expression across tissues and species. Epigenetics Chromatin [Internet]. 2018 Jun 29 [cited 2019 Oct 9];11. Available from: https://www.ncbi.nlm.nih.gov/pmc/articles/ PMC6025724/.

29. Yang X, Han H, De Carvalho DD, Lay FD, Jones PA, Liang G. Gene body methylation can alter gene expression and is a therapeutic target in cancer. Cancer Cell. 2014 Oct 13;26(4):577-90.

30. Paton VG, Shackelford JE, Krisans SK. Cloning and subcellular localization of hamster and rat isopentenyl diphosphate dimethylallyl diphosphate isomerase a PTS1 motif targets the enzyme to peroxisomes. J Biol Chem. 1997 Jul 25;272(30):18945-50

31. Wang C-C, Yen J-H, Cheng Y-C, Lin C-Y, Hsieh C-T, Gau R-J, et al. Polygala tenuifolia extract inhibits lipid accumulation in 3 T3-L1 adipocytes and highfat diet-induced obese mouse model and affects hepatic transcriptome and gut microbiota profiles. Food Nutr Res [Internet]. 2017 Oct 5 [cited 2019 Oct 14];61(1). Available from: https://www.ncbi.nlm.nih.gov/pmc/ articles/PMC5642193/.

32. Chatterjee TK, Idelman G, Blanco V, Blomkalns AL, Piegore MG, Weintraub DS, et al. Histone deacetylase 9 is a negative regulator of adipogenic differentiation. J Biol Chem. 2011 Aug 5:286(31):27836-47.

33. Akinyemiju T, Do AN, Patki A, Aslibekyan S, Zhi D, Hidalgo B, et al. Epigenome-wide association study of metabolic syndrome in AfricanAmerican adults. Clin Epigenetics. 2018;10:49. 
34. Cardona A, Day FR, Perry JRB, Loh M, Chu AY, Lehne B, et al. Epigenomewide association study of incident type 2 diabetes in a British Population: EPIC-Norfolk study. Diabetes. 2019 Sep;9. https://doi.org/10.2337/db18-0290.

35. Hidalgo B, Irvin MR, Sha J, Zhi D, Aslibekyan S, Absher D, et al. Epigenomewide association study of fasting measures of glucose, insulin, and HOMA-IR in the Genetics of Lipid Lowering Drugs and Diet Network study. Diabetes. 2014;63(2):801-7.

36. Kriebel J, Herder C, Rathmann W, Wahl S, Kunze S, Molnos S, et al. Association between DNA methylation in whole blood and measures of glucose metabolism: KORA F4 Study. PloS One. 2016;11(3):e0152314.

37. Buchmann J, Meyer C, Neschen S, Augustin R, Schmolz K, Kluge R, et al. Ablation of the cholesterol transporter adenosine triphosphate-binding cassette transporter G1 reduces adipose cell size and protects against dietinduced obesity. Endocrinology. 2007;148(4):1561-73.

38. Choromanska B, Mysliwiec P, Hady HR, Dadan J, Mysliwiec H, Bonda T, et al. The implication of adipocyte ATP-binding cassette A1 and G1 transporters in metabolic complications of obesity. J Physiol Pharmacol Off J Pol Physiol Soc. 2019 Feb;70(1). https://doi.org/10.26402/jpp.2019.1.14.

39. Mauldin JP, Nagelin MH, Wojcik AJ, Srinivasan S, Skaflen MD, Ayers CR, et al. Reduced expression of ATP-binding cassette transporter G1 increases cholesterol accumulation in macrophages of patients with type 2 diabetes mellitus. Circulation. 2008:117(21):2785-92.

40. Tavoosi Z, Moradi-Sardareh H, Saidijam M, Yadegarazari R, Borzuei S, Soltanian A, et al. Cholesterol transporters ABCA1 and ABCG1 gene expression in peripheral blood mononuclear cells in patients with metabolic syndrome. Cholesterol. 2015;2015:682904.

41. Arner P, Bernard S, Appelsved L, Fu K-Y, Andersson DP, Salehpour M, et al. Adipose lipid turnover and long-term changes in body weight. Nat Med. 2019;25(9):1385-9.

42. Aryee MJ, Jaffe AE, Corrada-Bravo H, Ladd-Acosta C, Feinberg AP, Hansen $K D$, et al. Minfi: a flexible and comprehensive bioconductor package for the analysis of Infinium DNA methylation microarrays. Bioinforma Oxf Engl. 2014;30(10):1363-9.

43. Xu Z, Niu L, Li L, Taylor JA. ENmix: a novel background correction method for Illumina HumanMethylation450 BeadChip. Nucleic Acids Res. 2016;44(3):e20.

44. Leek JT, Johnson WE, Parker HS, Jaffe AE, Storey JD. The sva package for removing batch effects and other unwanted variation in high-throughput experiments. Bioinforma Oxf Engl. 2012;28(6):882-3.

45. Ritchie ME, Phipson B, Wu D, Hu Y, Law CW, Shi W, et al. limma powers differential expression analyses for RNA-sequencing and microarray studies. Nucleic Acids Res. 2015;43(7):e47.

46. Du P, Zhang X, Huang C-C, Jafari N, Kibbe WA, Hou L, et al. Comparison of beta-value and $\mathrm{M}$-value methods for quantifying methylation levels by microarray analysis. BMC Bioinformatics. 2010;11(1):587.

47. Peters TJ, Buckley MJ, Statham AL, Pidsley R, Samaras K, Lord RV, et al. De novo identification of differentially methylated regions in the human genome. Epigenetics Chromatin. 2015 Jan 27;8(1):6.

\section{Publisher's Note}

Springer Nature remains neutral with regard to jurisdictional claims in published maps and institutional affiliations.

\section{Ready to submit your research? Choose BMC and benefit from}

- fast, convenient online submission

- thorough peer review by experienced researchers in your field

- rapid publication on acceptance

- support for research data, including large and complex data types

- gold Open Access which fosters wider collaboration and increased citations

- maximum visibility for your research: over $100 \mathrm{M}$ website views per year

At $\mathrm{BMC}$, research is always in progress.

Learn more biomedcentral.com/submissions 